\title{
Wetting in ternary mixtures-with and without amphiphiles
}

\author{
T. Schilling ${ }^{\text {a) }}$ and G. Gompper \\ Institut für Festkörperforschung, Forschungszentrum Jülich, 52425 Jülich, Germany
}

(Received 23 January 2002; accepted 1 July 2002)

\begin{abstract}
The interfacial wetting behavior of ternary fluid mixtures is investigated, both for systems where all components have isotropic interaction potentials, as well as for systems where one component is an amphiphile. The BEG model and the corresponding two-order-parameter Ginzburg-Landau model are employed for systems without amphiphiles. We calculate the global wetting phase diagram for nonamphiphilic mixtures. In the investigated range of interaction parameters, the wetting transitions are always continuous at three-phase coexistence. The critical behavior is found to be universal in some, nonuniversal in other parts of the phase diagram. For systems with amphiphiles, two additional interaction terms are taken into account. The first models the aggregation of amphiphilic molecules at the air-water interface, the second the formation of amphiphilic bilayers in water. We find that the first term leads to a reduction of the tension of the air-water interface, and favors wetting by the water-rich phase, while the second-bilayer-term leads to a reduction of the tension of the interface between the water-rich and amphiphile-rich phases. (c) 2002 American Institute of Physics. [DOI: 10.1063/1.1502242]
\end{abstract}

\section{INTRODUCTION}

The understanding of the wetting behavior of two-phase systems near a planar or curved wall, as well as of threephase system in thermal equilibrium has made enormous progress over the last 20 years. ${ }^{1-3}$ The theoretical work has mainly been focused on the case of two almost coexisting phases near a planar wall. Here, a rich phase behavior has been predicted from the mean-field analysis of the Ising ${ }^{4}$ and the corresponding Ginzburg-Landau models for two-phase coexistence, ${ }^{5}$ which was later confirmed and investigated in more detail. ${ }^{6-11}$

The coexistence of three fluid phases requires a system of at least three components if all phases are liquids, and at least two components if one of the phases is a vapor. In case that all the interactions between the different molecules are isotropic, such a system can be described theoretically very well by a Ginzburg-Landau model with squared-gradient approximation. For Ginzburg-Landau models with a single, scalar order parameter, the "middle" phase is then always found to wet the interface of the two other phases at threephase coexistence. ${ }^{12}$ Two scalar order parameters are needed in this case to describe the full wetting behavior, which includes the possibility of nonwetting of all three phases at coexistence. The second order parameter leads to the interesting possibility of nonuniversal wetting exponents for systems with short-ranged interactions already on the mean-field level. ${ }^{13-15}$ Models with two order parameters have also been used to investigate the effect of van der Waals interactions in binary fluid mixtures in coexistence with their vapor phase. ${ }^{16}$

The wetting behavior in ternary mixtures, which contain amphiphilic molecules, is of particular interest. ${ }^{17}$ In these systems, three homogeneous phases can coexist, which are

\footnotetext{
${ }^{a}$ Current address: FOM Institute for Atomic and Molecular Physics, Kruislaan 407, 1098 SJ Amsterdam, The Netherlands.
}

an oil-rich phase, a water-rich phase, and a microemulsion phase, which contains comparable amounts of oil and water and most of the surfactant. The surfactants reduce the interfacial tension of all three interfaces dramatically. The wetting behavior of the microemulsion phase at the oil-water interface is by now well understood. For short-chain surfactants, the microemulsion phase always wets the oil-water interface. For longer-chain surfactants, on the other hand, the microemulsion does not wet close to the phase-inversion temperature ${ }^{18}$ - where it contains exactly equal amounts of oil and water-while it wets when the upper or lower critical end points are approached, at which the microemulsion merges with the oil-rich or water-rich phase, respectively. ${ }^{19,20}$

This behavior can be understood theoretically very well on the basis of a Ginzburg-Landau model with a single, scalar order parameter, which is to be identified with the local concentration difference of oil and water. ${ }^{21}$ In this model, the nonwetting behavior is intimately connected to the oscillatory decay of the water-water (or oil-oil) correlation functions. Such correlations in the balanced system imply oscillations in the effective interface potential, and therefore lead to nonwetting. ${ }^{17,21-23}$ The oscillations are present for long-chain amphiphiles, but do not appear for short-chain amphiphiles. The connection between the decay of the bulk correlation function and the wetting behavior has been carefully investigated and confirmed experimentally. ${ }^{24}$

As the temperature is raised or lowered from the phaseinversion temperature, a critical end point is approached. In this case, the correlation function has to change from oscillatory to monotonic decay, and a wetting transition takes place. ${ }^{25-27}$

For amphiphilic systems, Ginzburg-Landau models with two scalar order parameters-the local concentration difference between oil and water, and the local surfactant concentration—have been employed to study correlations in 
the surfactant density and the corresponding scattering intensity. ${ }^{28-30}$ However, the surfactant concentration as second order parameter seems not to be essential for the wetting properties, since all experimentally observed effects can be captured by the single-order-parameter model discussed above.

The situation is different when the two-component fluid mixture of water and surfactant is considered in coexistence with its vapor (or air). The wetting behavior of such systems has been studied experimentally in Ref. 31. In this case, two order parameters are required to describe the interface. One order parameter is needed to describe the surfactant concentration of the fluid, and thus distinguishes the water-rich and the surfactant-rich phases, while the other order parameter is necessary to describe the mass density, and therefore to distinguish fluid and vapor phases. We want to investigate the wetting properties of this class of systems in more detail in this paper.

\section{THE BLUME-EMERY-GRIFFITHS MODEL}

\section{A. Spin-1 and Ginzburg-Landau models}

We employ a two-order-parameter Ginzburg-Landau (or van der Waals) model, which was first introduced by Blume, Emery, and Griffiths ${ }^{32}$ in order to describe mixtures of $\mathrm{He}^{3}$ and $\mathrm{He}^{4}$ at the $\lambda$ transition.

Consider a three component lattice gas, presented by a statistical variable $P_{i}^{\alpha}$ at each lattice site $i$ which is 1 if the site is occupied by a particle of species $\alpha$ and zero otherwise. All lattice sites are occupied. Nearest neighbors interact with each other with coupling strength $E^{\alpha \beta}$ and external fields $\mu^{\alpha}$ affect particles of the species $\alpha$. The Hamiltonian of the system is then

$$
\mathcal{H}=-\sum_{\alpha \beta} E^{\alpha \beta} \sum_{\langle i j\rangle} P_{i}^{\alpha} P_{j}^{\beta}-\sum_{\alpha} \mu_{\alpha} \sum_{i} P_{i}^{\alpha} .
$$

In the mean-field approximation, the free energy is a functional of the local densities

$$
\begin{aligned}
& \rho_{i}=\left\langle P_{i}^{A}\right\rangle, \\
& \psi_{i}=\left\langle P_{i}^{B}\right\rangle, \\
& 1-\psi_{i}-\rho_{i}=\left\langle P_{i}^{C}\right\rangle .
\end{aligned}
$$

The Ginzburg-Landau free-energy functional

$$
\begin{aligned}
F[\psi(\vec{x}), \rho(\vec{x})]= & \int d x^{3}\left\{-J\left(d \psi^{2}-\frac{1}{2}(\nabla \psi)^{2}\right)\right. \\
& -K\left(d \rho^{2}-\frac{1}{2}(\nabla \rho)^{2}\right)-C(2 d \psi \rho-\nabla \psi \nabla \rho) \\
& -\mu_{\psi} \psi-\mu_{\rho} \rho+T(\psi \ln \psi+\rho \ln \rho \\
& +(1-\psi-\rho) \ln (1-\psi-\rho))\}
\end{aligned}
$$

is then derived in the continuum limit, where $d$ is the space dimension and

$$
\begin{aligned}
& J=E^{B B}+E^{C C}-2 E^{B C}, \\
& K=E^{A A}+E^{C C}-2 E^{A C}, \\
& C=E^{C C}+E^{A B}-E^{A C}-E^{C B},
\end{aligned}
$$

$$
\begin{aligned}
& \mu_{\psi}=\mu_{B}-\mu_{C}-2 \mathrm{~d} E^{C C}+2 \mathrm{~d} E^{B C}, \\
& \mu_{\rho}=\mu_{A}-\mu_{C}-2 \mathrm{~d} E^{C C}+2 \mathrm{~d} E^{A C} .
\end{aligned}
$$

There are three high-symmetry cases. When the system is invariant under exchange of $A$ and $B$ molecules, then $J$ $=K$. For a system which is invariant under an exchange of $A$ and $C$ molecules, $K=2 C$, and similarly, for a system which is invariant under an exchange of $B$ and $C$ molecules, $J$ $=2 C$. See Appendix for a more detailed discussion.

The model is often formulated in magnetic language instead, with a spin variable $S_{i}$ at each site, which takes the values 1,0 , and -1 . In this case, the Hamiltonian reads

$$
\begin{aligned}
\mathcal{H}= & -\sum_{\langle i j\rangle}\left[\widetilde{J} S_{i} S_{j}+\widetilde{C}\left(S_{i} S_{j}^{2}+S_{i}^{2} S_{j}\right)+\widetilde{K} S_{i}^{2} S_{j}^{2}\right] \\
& -\sum_{i}\left(H S_{i}+\Delta S_{i}^{2}\right) .
\end{aligned}
$$

The two models are easily mapped onto each other with the relations

$$
\begin{aligned}
& P_{i}^{A}=-\frac{1}{2} S_{i}\left(1-S_{i}\right), \\
& P_{i}^{B}=\frac{1}{2} S_{i}\left(1+S_{i}\right), \\
& P_{i}^{C}=1-S_{i}^{2},
\end{aligned}
$$

and

$$
\begin{aligned}
& 4 \widetilde{J}=J+K-2 C, \\
& 4 \widetilde{K}=J+K+2 C, \\
& 4 \widetilde{C}=J-K, \\
& 2 H=\mu_{\psi}-\mu_{\rho}, \\
& 2 \Delta=\mu_{\psi}+\mu_{\rho} .
\end{aligned}
$$

The global bulk phase diagram of the Blume-EmeryGriffiths (BEG) model is described in the work of Furman, Dattagupta, and Griffiths. ${ }^{33}$ Here we sketch only those features of the bulk behavior, which are relevant for wetting processes. Figure 1 shows coexistence regions in the composition triangle and coexistence lines in the $\left(\mu_{\psi} \mu_{\rho}\right)$ plane for two sets of coupling parameters $J K C$ with different values of $J$. In the composition triangle three two-phase regions meet in one three-phase region, which grows with increasing $J$. Thus, $J$ controls the size of the mixing gap between the $B$ and $C$ phase. With decreasing $J$ the critical point at the end of the $B C$ coexistence line in the $\left(\mu_{\psi} \mu_{\rho}\right)$ picture moves into the three-phase point and vanishes there, which means that the three-phase triangle in the corresponding composition picture degenerates into a line. The equivalent happens to the $A C$-mixing gap for small $K$, as the model is symmetric in $J$ and $K$, and the $A B$-mixing gap vanishes for sufficiently large $C$. 

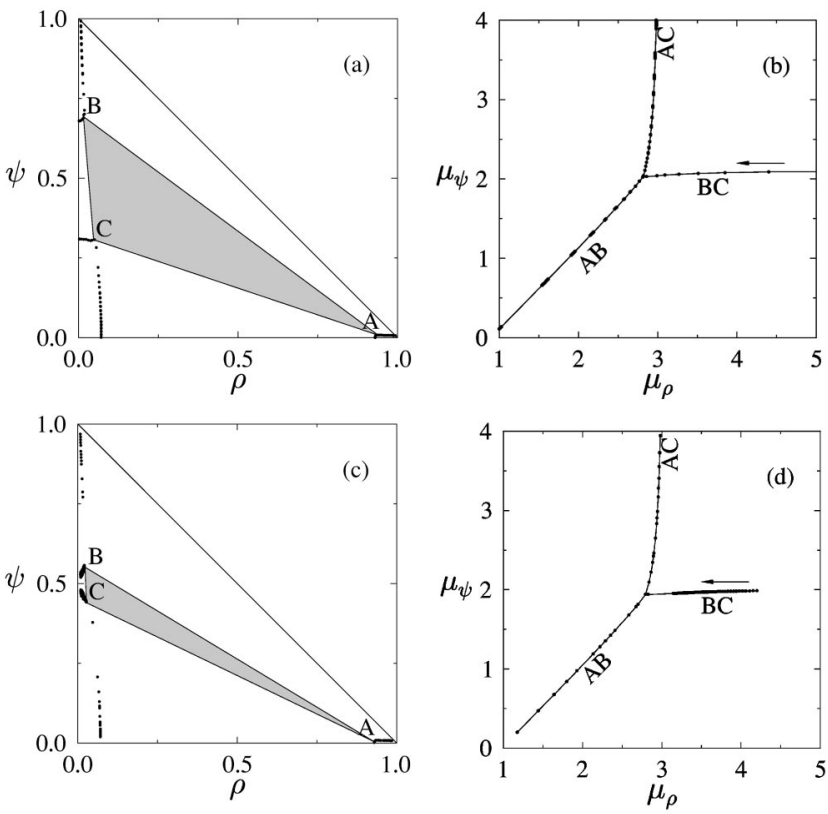

FIG. 1. Dependence of the bulk phase diagram on the interaction parameter $J$, for $K=1, C=0$, with (a), (b) $J=0.7$, and (c), (d) $J=0.665$. Decreasing $J$ moves the critical point at the end of the $B C$-coexistence line into the three-phase coexistence point (b), (d). The three-phase triangle degenerates into a line (c).

Combination of these observations yields Fig. 2, a schematic view of the region in parameter space, in which three bulk phases coexist. The surfaces of this region consist of critical end points, at which two of the three liquids become identical.

\section{B. Approach to wetting}

We employ the following procedure to investigate the wetting properties of the model. For a given set of parameters within the three-phase region, we first determine the bulk densities numerically and then minimize the free-energy functional (3) with respect to all density profiles $\psi(x), \rho(x)$ with the bulk values as boundary conditions. In order to find the profiles, we discretize space in an interval much larger

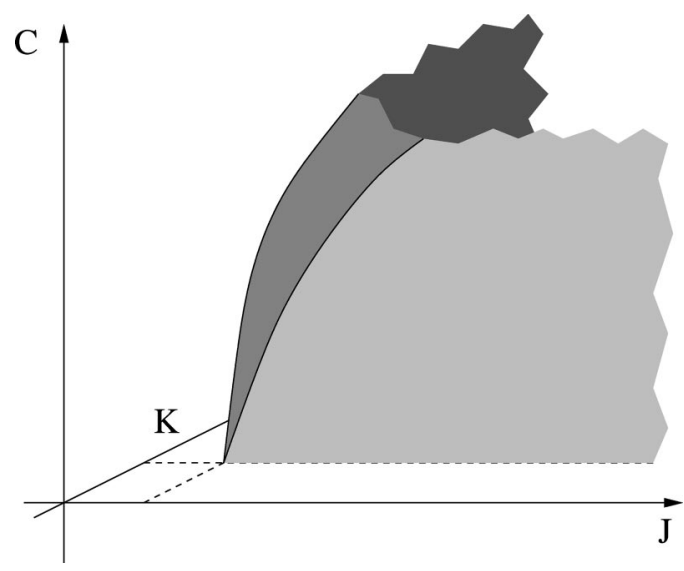

FIG. 2. Schematic view of the three-phase region in parameter space. The surfaces of the three-phase region consist of critical end points at which two of the coexisting phase merge.

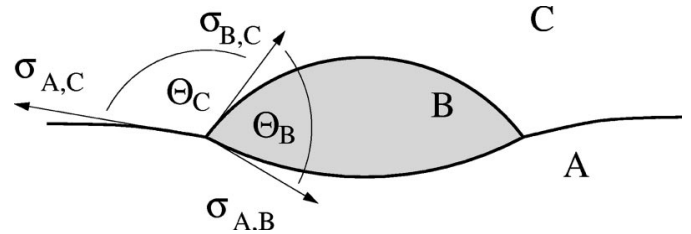

FIG. 3. Definitions of the contact angles of a fluid droplet at a fluid-fluid interface.

than the interfacial width and then minimize $F\left[\psi\left(x_{i}\right), \rho\left(x_{i}\right)\right]$ with respect to the variables $\left(\psi\left(x_{i}\right), \rho\left(x_{i}\right)\right)$ under the boundary condition that two points at each end of the profile are fixed at bulk values. The difference between the free energy of the interfacial profile, which minimizes Eq. (3), and the free energy of a bulk phase is the interfacial tension $\sigma$. From the interfacial tensions of the three interfaces follow the contact angles

$$
\cos \left(\Theta_{B}\right)=-\frac{\sigma_{A, B}^{2}+\sigma_{B, C}^{2}-\sigma_{A, C}^{2}}{2 \sigma_{A, B} \sigma_{B, C}},
$$

$$
\cos \left(\Theta_{C}\right)=-\frac{\sigma_{A, B}^{2}+\sigma_{A, C}^{2}-\sigma_{B, C}^{2}}{2 \sigma_{A, B} \sigma_{A, C}},
$$

compare Fig. 3. In order to characterize the wetting transitions, we compare the critical exponent of the contact angle and the film thickness growth rate to analytical results for an approximate effective interfacial potential.

\section{Effective interfacial potential}

If an interface is not wet, there is yet a microscopically thin film spread at it. In order to describe critical wetting transitions, we discuss the effective potential ${ }^{1-3}$

$$
\omega(l)=F[l, T, \mu]-l f_{\mathrm{Vol}}-\sigma_{A, B}-\sigma_{B, C},
$$

between the two interfaces which bound this film, where $l$ is the film thickness. If the interface is wet, $\omega(l)$ has its minimum at infinite $l$, if it is not wet, there is a minimum at finite $l$.

We assume $l$ to be large enough for the densities to relax into their bulk values at each interface as if they were independent. Small deviations $\delta \psi(x), \delta \rho(x)$ from the bulk values $\bar{\psi}(x), \bar{\rho}(x)$ produce a change in free energy 


$$
\begin{aligned}
\Delta F[\delta \psi(x), \delta \rho(x)]= & \int d x\left\{-J\left(d \delta \psi^{2}-\frac{1}{2}(\nabla \delta \psi)^{2}\right)-K\left(d \delta \rho^{2}-\frac{1}{2}(\nabla \delta \rho)^{2}\right)-C(2 d \delta \psi \delta \rho-\nabla \delta \psi \nabla \delta \rho)\right. \\
& \left.+T\left(\frac{\delta \psi^{2}}{2 \bar{\psi}}+\frac{\delta \rho^{2}}{2 \bar{\rho}}+\frac{(\delta \psi+\delta \rho)^{2}}{2(1-\bar{\psi}-\bar{\rho})}\right)\right\} .
\end{aligned}
$$

The solution of the Euler-Lagrange equations derived from Eq. (11) for an ansatz of exponential decays

$$
\delta \psi=\sum_{i=1}^{2} a_{i} e^{-\lambda_{i} x}, \quad \delta \rho=\sum_{i=1}^{2} b_{i} e^{-\lambda_{i} x},
$$

yields the decay exponents

$$
\lambda_{1,2}^{2}=\frac{K Q+J R-2 C S \mp \sqrt{(K Q-J R)^{2}-4\left[S^{2} J K+C^{2} Q R-C S(K Q+J R)\right]}}{2\left(J K-C^{2}\right)}
$$

with

$$
\begin{aligned}
& Q=-2 d J+\frac{T}{\bar{\psi}}+\frac{T}{1-\bar{\psi}-\bar{\rho}}, \\
& R=-2 d K+\frac{T}{\bar{\rho}}+\frac{T}{1-\bar{\psi}-\bar{\rho}}, \\
& S=-2 d C+\frac{T}{1-\bar{\psi}-\bar{\rho}} .
\end{aligned}
$$

From the ansatz (12) follows the form of the interfacial potential:

$$
\begin{aligned}
\omega(l) & =r e^{-\lambda_{1} l}+s e^{-\left(\lambda_{1}+\lambda_{2}\right) l}+t e^{-\lambda_{2} l}+u e^{-2 \lambda_{1} l}+\cdots \\
& \equiv A e^{-\alpha l}+B e^{-\beta l}+\cdots .
\end{aligned}
$$

We consider only the two terms of leading order

$$
\begin{aligned}
& \alpha=\lambda_{1}, \quad \beta=\lambda_{2} \quad \text { if } \lambda_{1}<\lambda_{2}<2 \lambda_{1}, \\
& \alpha=\lambda_{1}, \quad \beta=2 \lambda_{1} \quad \text { if } \lambda_{1}<2 \lambda_{1}<\lambda_{2} .
\end{aligned}
$$

The prefactors $A$ and $B$ depend on the interaction parameters, the bulk densities, and the decay exponents $\lambda_{1,2}$. The effective interface potential $\omega(l)$ has its minimum at

$$
l_{0}=\frac{1}{\beta-\alpha} \ln \left(\frac{B \beta}{A \alpha}\right),
$$

with $A<0, B>0$ in order to start out from a non-wetting situation, i.e., a minimum of $\omega(l)$ at finite $l$. As $A$ goes to zero on approach of the wetting transition, the film thickness grows with an exponent $1 /(\beta-\alpha) \equiv a_{l}$. The contact angle, written as $\cos \left(\Theta_{B}\right)$, follows the power law

$$
\cos \left(\Theta_{B}\right) \approx 1-\frac{\omega\left(l_{0}\right)}{\sigma_{B, C}}=: 1-A^{\gamma}
$$

with $\gamma=\beta /(\beta-\alpha)$.

\section{RESULTS FOR SYSTEMS WITH ISOTROPIC INTERACTIONS}

\section{A. Global wetting phase diagram}

Figure 4 shows a cut through the three-phase region at $C=1$. The solid lines are contact angle isolines for $\Theta_{B}$ and $\Theta_{C}$. The dashed lines mark the surface of the three-phase region. The solid thick lines are $0^{\circ}$ and $180^{\circ}$ isolines. For small $J$, i.e., close to the critical point of the $B C$ mixture, $\Theta_{B}=0^{\circ}$ and $\Theta_{C}=180^{\circ}$ - the $B$ phase wets the $A C$ interface. For small $K$ the $A$ phase wets the $B C$ interface. At $J=K$ (triangle) there is no wetting because $\sigma_{A, C}=\sigma_{B, C}$ all the way to the critical end point. Wetting close to a critical end point of the mixture-where the interfacial tension between the two phases which become critical goes to zero, while the interfacial tensions of these phases with the spectator phase approach the same finite value-is often referred to as "Cahn" wetting. ${ }^{34}$

Figure 5 shows cuts at $C=1.5$ and $C=2$ for comparison. The general structure remains the same, but the wetting regions grow larger due to the bent shape of the three-phase surface with respect to $C$.

The wetting behavior for an orthogonal cut through the parameter space, at constant interaction strength $J$, is shown in Fig. 6. This cut shows in particular that the wetting tran-
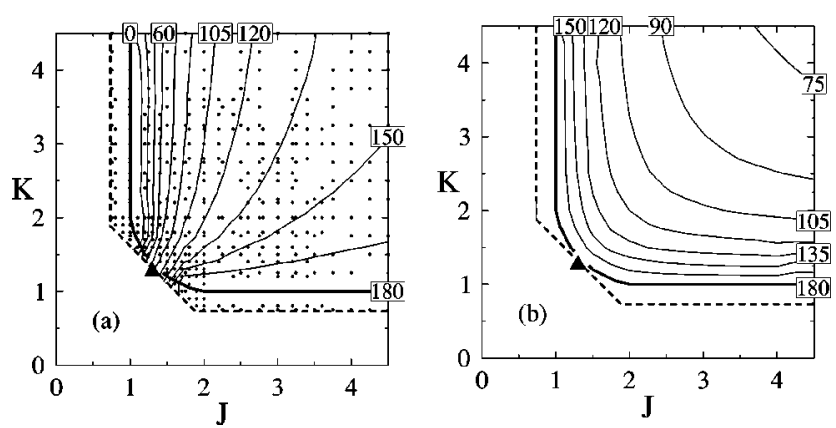

FIG. 4. Contact angles (a) $\Theta_{B}$ and (b) $\Theta_{C}$ for a cut through the three-phase region at $C=1$. Dots mark parameters for which numerical data was taken, solid lines are contact angle isolines, which were interpolated from the data, the dashed line marks the boundary of the coexistence region, and the thick solid lines are $0^{\circ}$ and $180^{\circ}$ isolines. 

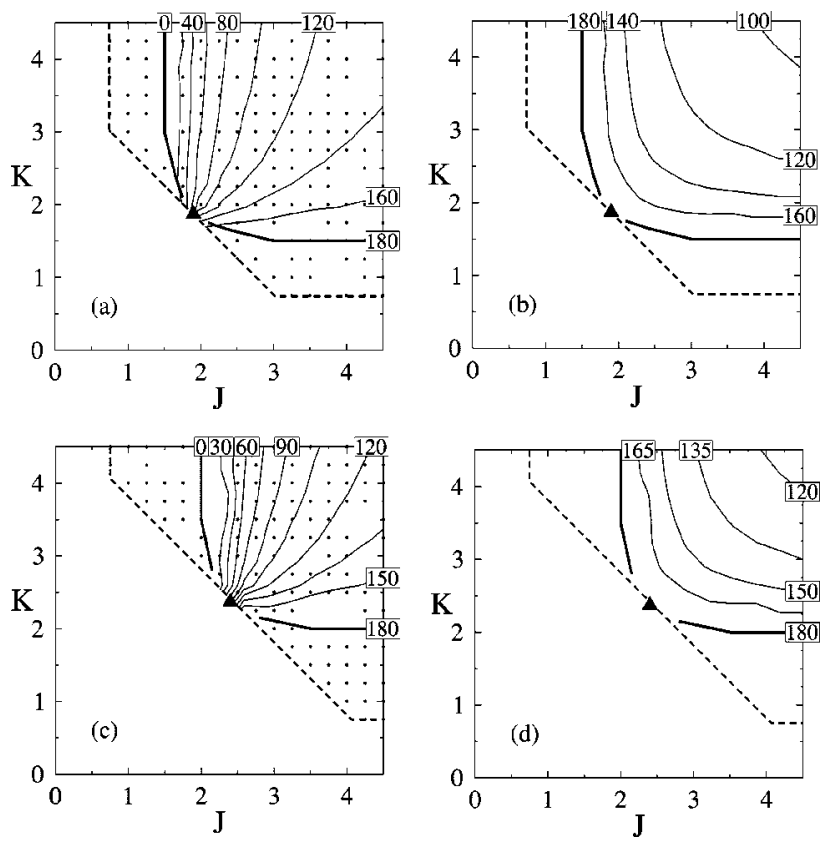

FIG. 5. Contact angles (a), (c) $\Theta_{B}$ and (b), (d) $\Theta_{C}$ for $C=1.5$ (a), (b) and for $C=2$ (c), (d). Dots mark parameters for which numerical data was taken, solid lines are contact angle isolines, which were interpolated from the data, the dashed line marks the boundary of the coexistence region, and the thick solid lines are $0^{\circ}$ and $180^{\circ}$ isolines.

sition of the $A B$ interface by the $C$ phase over a large range of $K$ values occurs very close to the $C=0$ plane.

From these different cuts through the parameter space, the full wetting diagram can now be constructed, see Fig. 7, where the boundaries of the wetting regions are drawn into the three-phase region. Additionally to "Cahn" wetting, which occurs when the wetting surface comes very close to the surface of critical end points, wetting transitions also occur further away from these "critical surfaces." This is a different type of wetting, which occurs for $C<0$ because $\sigma_{A, B}$ gets very large. The same effect occurs when $\sigma_{A, C}$ or $\sigma_{B, C}$ gets large (by appropriate choice of $K+C$ or $J+C$, respectively). Considering the evolution of the profiles on approach of the wetting transitions, we find all wetting tran-
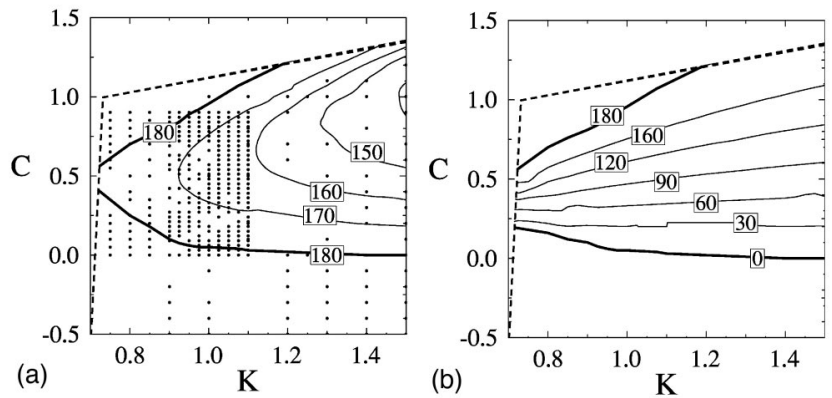

FIG. 6. Contact angles (a) $\Theta_{B}$ and (b) $\Theta_{C}$ for $J=2$. Dots mark parameters for which numerical data was taken, solid lines are contact-angle isolines, which were interpolated from the data, the dashed line marks the boundary of the coexistence region, and the thick solid lines are $0^{\circ}$ and $180^{\circ}$ isolines. The contact-angle isolines do not converge at $K \simeq 0.75$ to a single point (as they should) due to the interpolation algorithm.

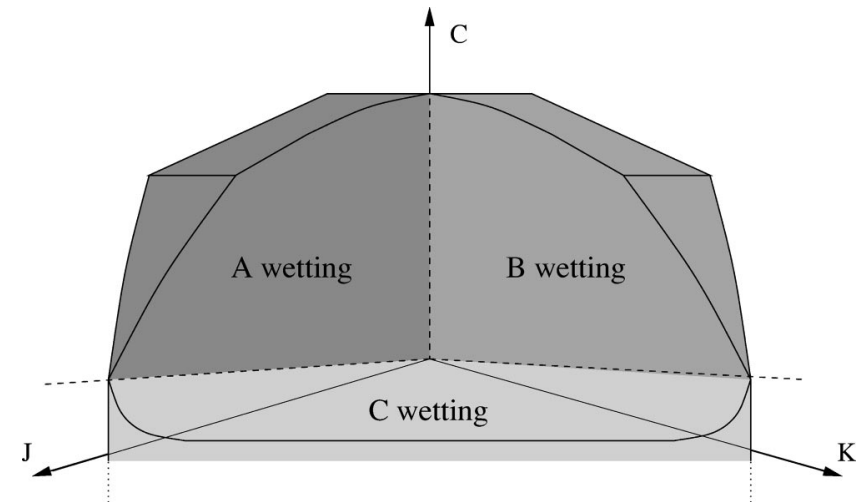

FIG. 7. Sketch of the full wetting diagram. For $C>0$, the surfaces of the three-phase region are covered by wetting regions, which can be "Cahntype" close to the dashed lines, and "non-Cahn" otherwise. For $C \leq 0$ we find a "non-Cahn" wetting behavior, in which the $C$ phase wets the $A B$ interface far from the critical end point. Along the dashed lines, no wetting transitions occur, because two of the interfacial tensions remain equal all the way to the critical end point.

sitions to be continuous in the investigated range of interaction parameters.

\section{B. Critical exponent of the contact angle}

The critical properties obtained from the numerical calculation can be compared with predictions by the effective interfacial potential approach. Figure 8 shows the film thickness $l$ and the contact angle $\Theta_{B}$ versus the distance $j \equiv(J$ $\left.-J_{W}\right) / J_{W}$ from the $B$-wetting transition, where $J_{W}$ is the transition point. The circles are data from the numerically optimized profiles, the solid lines are results from the approximation of the interfacial potential. The film thicknesswhich is defined as the distance between the two inflection points of the order parameter profile-grows like $\ln (j)$. Close to the transition the interfacial potential approach produces a good approximation. For $j>0.06$ it deviates from the data, because the interfaces are too close to still be regarded as independent. 1- $\cos \left(\Theta_{B}\right)$ follows a power law, the exponent of which is also well reproduced by the approximation.

Figure 9 shows the decay exponents $\lambda_{1,2}$ and the exponent of the contact angle $\gamma$ (crosses) and the film thickness-
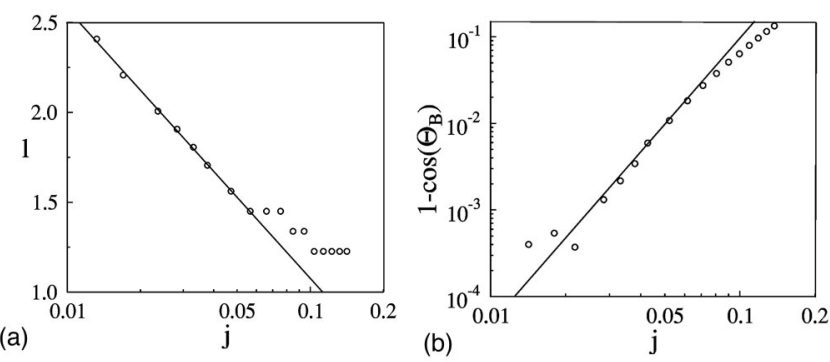

FIG. 8. (a) Film thickness $l$ and (b) contact angle $\Theta_{B}$ on approach of a wetting transition, with $j \equiv\left(J-J_{W}\right) / J_{W}$. Data from numerical calculations (circles) and approximation from effective interface potential $\omega(l)$; see Eqs. (17) and (18) (solid lines), respectively. The parameters are $K=2.2, C=1$, $J_{W}=1.06, a_{l}=-0.69$, and $\gamma=3.4$. 

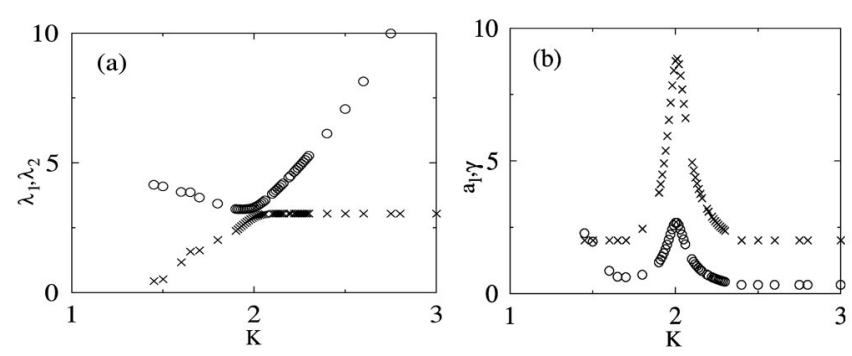

FIG. 9. (a) Decay exponents $\lambda_{1,2}$. (b) Critical exponent of contact angle, $\gamma$ (crosses), and film thickness growth law, $a_{l}$ (circles), along a line of wetting transitions with $C=1.0$ and $J \simeq 1.0$ (compare Fig. 4). At $K \simeq 2 J \simeq 2 C$ the solutions of Eq. (13) are almost degenerate. Therefore $\gamma$ and $a_{l}$ become very large.

growth rate $a_{l}$ (circles) from the effective interfacial potential calculations along a line of wetting transitions with $C$ $=1.0$ and $J \simeq 1.0$ (compare Fig. 4). For most of the phase diagram, $\lambda_{2}>2 \lambda_{1}$ and therefore $\gamma=2$ and $a_{l}=1 / \lambda_{1}$. At $K$ $\simeq 2 J \simeq 2 C$ the exponents deviate from this behavior and grow very large (they diverge for infinite $J$ ). The symmetry which causes the close approach of the two eigenvalues $\lambda_{1}$ and $\lambda_{2}$ is

$$
\rho_{B}=1-\rho_{B}-\psi_{B},
$$

where $\rho_{B}$ denotes the $A$ particle density within the $B$-rich middle phase. The densities of $A$ and $C$ particles within the middle phase are identical at this point. Substitution of Eq. (19) and $K=2 J=2 C$ into Eq. (13) yields

$$
\lambda_{1,2}^{2}=\frac{1}{J}\left(Q \mp \frac{2}{\psi_{B}}\right) .
$$

For growing $J$ the $B$-rich phase becomes purer and the bulk densities $\psi_{B}$ and $\rho_{B}$ move towards 1 and 0 , respectively. Therefore, $Q$ grows very large while $\psi_{B}$ approaches unity. The solutions $\lambda_{1,2}$ become more and more similar and thus $\gamma$ and $a_{l}$ become very large, but they do not diverge unless $J \rightarrow \infty$.

\section{Discussion}

On the basis of our results, we can now compare the wetting behavior of the ternary system with the wetting behavior of a two-component system near a wall. Indeed, the spectator phase in wetting transitions of three-component systems near a critical end point (CEP) is often described theoretically by an inert wall. ${ }^{35,36}$ In this case, the wetting phase diagram depends on the strength of the interactions at the wall. ${ }^{5}$ If these interactions are much stronger than in the bulk, a surface transition occurs above the bulk critical temperature, where one component is enriched in a microscopically thin surface layer, while the bulk remains homogeneously mixed.

The wetting phase diagram in the opposite case of sufficiently weak surface interactions is shown in Fig. 10. This is expected to be the generic situation. It shows that for small surface fields, a critical wetting transition occurs near the bulk critical point, while for stronger surface fields, the wetting transition becomes first order. No wetting transition oc-

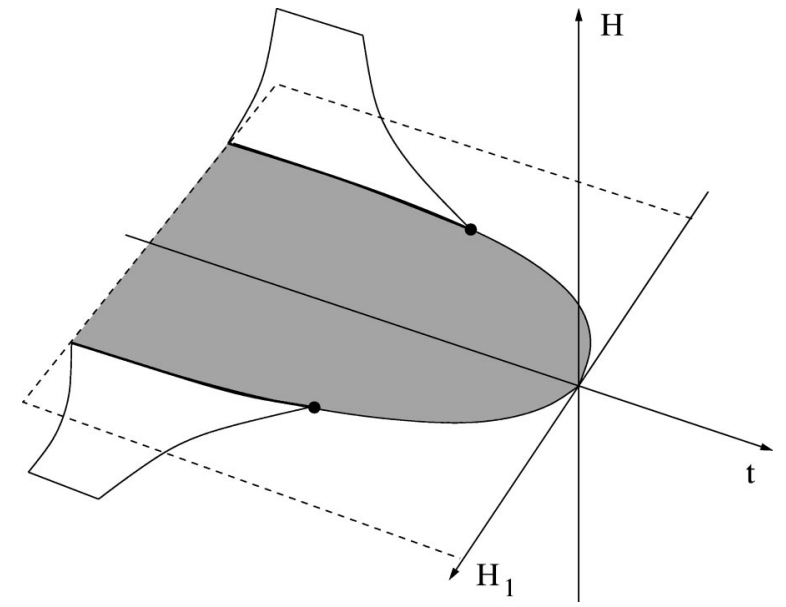

FIG. 10. Wetting phase diagram of a two-component mixture near a wall. Here, $t=\left(T-T_{c}\right) / T_{c}$ is the reduced temperature, $H$ is a bulk field proportional to the chemical potential difference between the two components [compare Eq. (7)], and $H_{1}$ is the corresponding surface field. In the shaded region of the coexistence plane $(H=0)$, neither the $A$ rich nor the $B$ rich phase wets the wall. The two wings extending into the region $H \neq 0$ are prewetting surfaces. Redrawn from Ref. 5.

curs in the absence of a surface field, because both phases have exactly the same surface free energy in this case.

In our wetting phase diagram of Figs. 4, 5, and 7, there are three lines at which no wetting transition occurs. These are the lines, where two of the three interfacial tensions are identical, so that again no wetting transition can occur by symmetry. At constant $C$, the wetting phase diagram in the vicinity of symmetry point at $J=K$ then indeed resembles the diagram of Nakanishi and Fisher ${ }^{5}$ very closely. Firstorder transitions have not been found in the investigated part of the parameter space, so that prewetting wings are absent in our phase diagram. The situation is very similar for the other two lines, where $\sigma_{A, B}=\sigma_{B, C}$ and $\sigma_{A, B}=\sigma_{A, C}$, respectively.

Two scenarios are now possible. First, wetting at threephase coexistence is indeed equivalent to wetting of a wall at two-phase coexistence. In this case, the analogy with the phase diagram of Nakanishi and Fisher ${ }^{5}$ implies that the wetting transitions in the ternary system will become first order sufficiently far from the symmetry lines. This has not been observed in the investigated range of interaction parameters, but could occur for even larger interaction strengths. Second, the fact that we have not been able to find first-order wetting transitions could indicate that this equivalence does not always hold.

\section{TERNARY MIXTURES WITH AMPHIPHILES}

We investigate the wetting properties of a mixture of water $(W)$ and amphiphile $(A)$ at the interface to their vapor phase or air $(G)$. Amphiphilic properties are incorporated into the model by two additional interactions, 


$$
\begin{aligned}
\mathcal{H}= & -\sum_{\alpha} \sum_{i} \mu^{\alpha} P_{i}^{\alpha}-\sum_{\alpha, \beta} \sum_{\langle i, j\rangle} E^{\alpha \beta} P_{i}^{\alpha} P_{j}^{\beta} \\
& -\sum_{\langle i, j, k\rangle} 2 L_{1} P_{i}^{G} P_{j}^{A} P_{k}^{W} \\
& -\sum_{\langle i, j, k, l\rangle} L_{4} P_{i}^{W} P_{j}^{A} P_{k}^{A} P_{l}^{W} .
\end{aligned}
$$

The three-particle interaction models the amphiphiles' property to assemble at the water-air interface. The four-particle interaction accounts for its tendency to self-assemble within the water phase. Under the same approximations as before, and with

$$
\begin{aligned}
& \rho_{i}=\left\langle P_{i}^{W}\right\rangle, \\
& \psi_{i}=\left\langle P_{i}^{A}\right\rangle, \\
& 1-\psi_{i}-\rho_{i}=\left\langle P_{i}^{G}\right\rangle,
\end{aligned}
$$

follows the free-energy functional

$$
\begin{aligned}
F= & \int d x^{3}\left\{-\mu_{\psi} \psi-\mu_{\rho} \rho-J\left(d \psi^{2}-\frac{1}{2}(\nabla \psi)^{2}\right)-K\left(d \rho^{2}-\frac{1}{2}(\boldsymbol{\nabla} \rho)^{2}\right)-\left(C+2 L_{1}\right)(2 d \psi \rho-\nabla \psi \nabla \rho)\right. \\
& +2 L_{1}\left[d\left(\psi^{2} \rho+\rho^{2} \psi\right)-\frac{1}{2} \rho\left((\nabla \psi)^{2}-\frac{1}{12}(\Delta \psi)^{2}\right)-2 \psi\left((\boldsymbol{\nabla} \rho)^{2}-\frac{1}{3}(\Delta \rho)^{2}\right)-\boldsymbol{\nabla} \psi \boldsymbol{\nabla} \rho\left(\rho+\frac{5}{2} \psi\right)\right] \\
& -L_{4}\left[d \psi^{2} \rho^{2}-\frac{1}{2} \rho^{2}\left((\boldsymbol{\nabla} \psi)^{2}-\frac{1}{12}(\Delta \psi)^{2}\right)-\frac{9}{2} \psi^{2}\left((\boldsymbol{\nabla} \rho)^{2}-\frac{3}{4}(\Delta \rho)^{2}\right)-5 \psi \rho \nabla \psi \nabla \rho\right] \\
& \left.+T\left(\frac{\psi}{N_{A}} \ln \frac{\psi}{N_{A}}+\rho \ln \rho+(1-\psi-\rho) \ln (1-\psi-\rho)\right)\right\}
\end{aligned}
$$

where $N_{A}$ is the length of the amphiphile. In the free-energy functional (23), we include in the expansion of the interaction terms contributions up to fourth power in the densities and up to four derivatives. However, all terms have been neglected, which contain products of derivatives of $\psi$ and $\rho$ and have a total of four derivatives, since such terms lead to numerical instabilities. We will demonstrate below that the interfacial profiles depend qualitatively on $L_{1}$ and $L_{4}$ as expected, so that our model (23) captures the essential physics of these systems.

We employ the same numerical methods for the determination of the interfacial densities as before and are therefore restricted to homogeneous bulk phases. The stability of the bulk phases against spatial variations of the order parameters is analyzed within the Ornstein-Zernike approximation. We find the disordered phases to be stable approximately for $L_{4} \lesssim L_{1} / 2$.

\section{RESULTS FOR AMPHIPHILIC MIXTURES}

The presence of amphiphilic molecules alters the wetting properties of a mixture in two ways: The bulk diagram changes because of changes in solubility and the interfacial structure changes because of the self-assembling property of the amphiphilic molecules.

\section{A. Bulk properties}

The "length" $N_{A}$ of the amphiphiles reduces the influence of the entropic term in $\psi$ and therefore has the same effect on the bulk diagram as a stronger coupling $J$. It opens the mixing gap between the amphiphile-rich and the vapor phase. $L_{1}$ couples $\psi$ and $\rho$ and has a similar effect as $C$. It reduces the mixing gap between water and amphiphile-rich phase. $L_{4}$ does the same in principle, but it does not have any influence on the bulk diagram in the parameter range we have chosen for our investigation of the wetting properties. All the results presented below have been obtained for $N_{A}$ $=1$.

\section{B. Wetting properties}

The wetting properties of the amphiphilic system as a function of the amphiphile strengths $L_{1}$ and $L_{4}$ are qualitatively different for $J>K$ and $J<K$.

We consider first the case $J>K$. Figure 11(a) shows profiles of the amphiphile density $\psi$ at the water-air interface in dependence of the amphiphilicity $L_{1}$. Amphiphile accumulates at the interface with increasing $L_{1}$. However, this effect is small, and leads to only a very weak reduction of the interfacial tension $\sigma_{W, G}$; see Fig. 11(b). More dramatic is the increase of the content of amphiphile in the bulk water phase with increasing $L_{1}$. This increase indicates an ap-
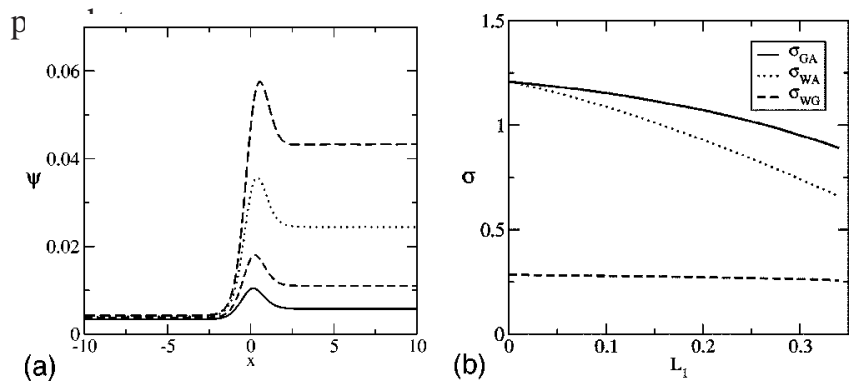

FIG. 11. (a) Profiles of amphiphile density $\psi$ at the air-water interface (with water-rich phase located at $x>0$ ) for varying amphiphilicity $L_{1}=0.1-0.4$ (solid-long-dashed curve), with $J=2, K=1, C=0.5$, and $L_{4}=0$, and (b) corresponding interfacial tensions. The accumulation of amphiphilic molecules at the interface reduces $\sigma_{W, G}$ only slightly, but the increase of amphiphile in the bulk water phase produces a large decrease in $\sigma_{W, A}$. 

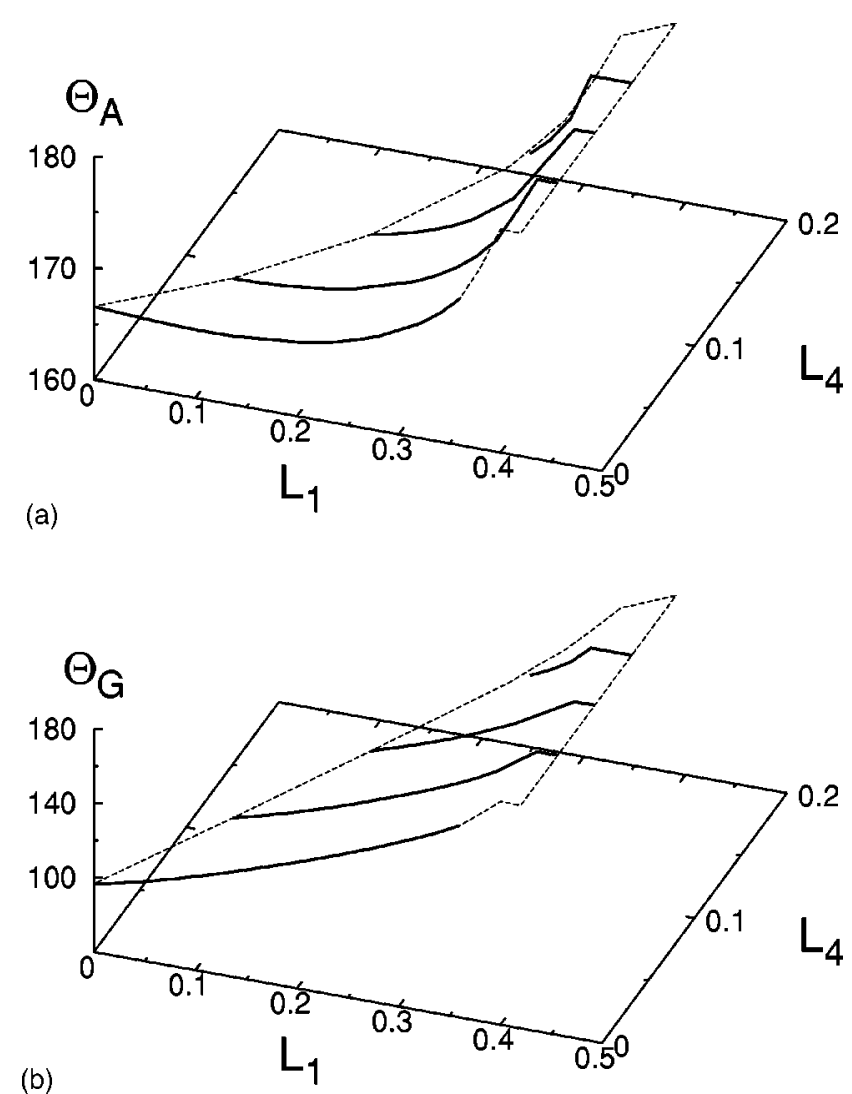

FIG. 12. Contact angles (a) of the amphiphile-rich phase, $\Theta_{A}$, and (b) of the vapor phase, $\Theta_{G}$, in amphiphilic mixtures with $J>K$, as a function of the two interaction parameters $L_{1}$ and $L_{4}$ characterizing the amphiphile strength. Both contact angles grow to $180^{\circ}$ with growing amphiphilicity. A transition to wetting of the $A G$ interface by a water film is induced with increasing $L_{1}$. The parameters are $J=2, K=1$, and $C=0.5$.

the critical point, where the water-rich and the amphiphilerich phases merge, and therefore leads to a pronounced reduction of the interfacial tension $\sigma_{W, A}$ between these two phases; compare Fig. 11(b). Thus, increasing $L_{1}$ drives the system towards a wetting transition of the water-air interface by the amphiphile-rich phase.

Our data for the contact angles within the amphiphilerich phase $\left(\Theta_{A}\right)$ and within the vapor phase $\left(\Theta_{G}\right)$ versus $L_{1}$ and $L_{4}$ are shown in Fig. 12. Both contact angles grow
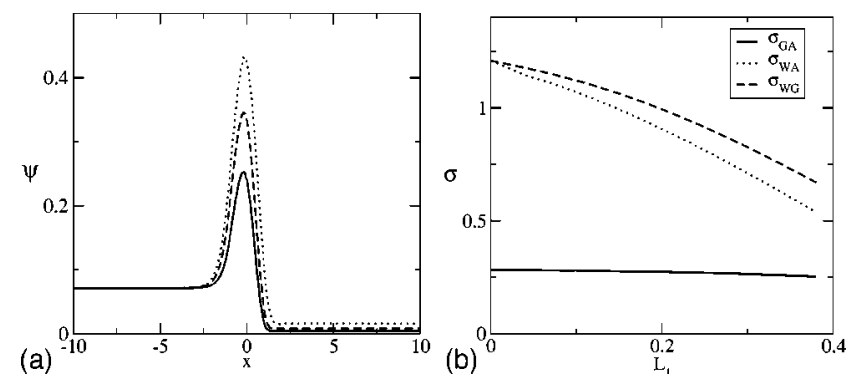

FIG. 13. (a) Profiles of amphiphile density $\psi$ at the air-water interface (with water-rich phase located at $x>0$ ) for varying amphiphilicity $L_{1}=0.1-0.3$ (solid-dotted curve), with $J=1, K=2, C=0.5$, and $L_{4}=0$, and (b) corresponding interfacial tensions. Note the different scale of the density axis compared to Fig. 11. Increasing $L_{1}$ leads to a strong accumulation of amphiphile at the interface of water and air. $\sigma_{W, G}$ decreases strongly.
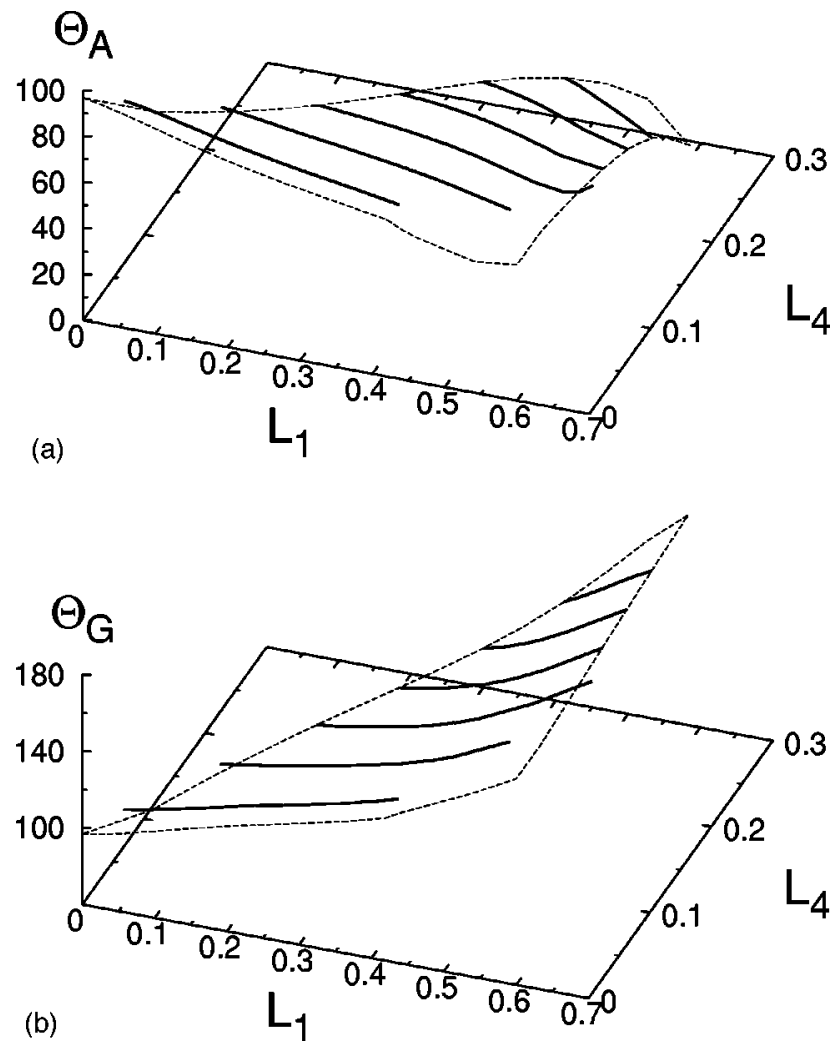

FIG. 14. Contact angles (a) of the amphiphile-rich phase, $\Theta_{A}$, and (b) of the vapor phase, $\Theta_{G}$, in amphiphilic mixtures with $J<K$, as a function of the two interaction parameters $L_{1}$ and $L_{4}$ characterizing the amphiphile strength. Wetting of the $W G$ interface by an amphiphile-rich film only occurs at finite $L_{4}>0$. The parameters are $J=1, K=2$, and $C=0.5$.

weakly with $L_{4}$ and strongly with $L_{1}$, until water wets the $A G$ interface. The reason the water phase wets the $A G$ interface and not the amphiphile-rich phase the $W G$ interface is that for $J>K, \sigma_{W, G}<\sigma_{A, G}$. This agrees with experimental findings by Kahlweit and Busse. ${ }^{31}$ A more detailed comparison will be made in Sec. VII below.

In the opposite case $J<K$, the dependence of wetting behavior on $L_{1}$ and $L_{4}$ is quite different. Now, increasing $L_{1}$ leads to a strong accumulation of amphiphile at the interface of water and air, as well as at the interface of water and amphiphile-rich phase, as shown in Fig. 13(a). Therefore, the interfacial tensions $\sigma_{W, G}$ and $\sigma_{W, A}$ both show a pronounced decrease with increasing $L_{1}$, while $\sigma_{A, G}$ shows only a weak decrease; compare Fig. 13(b).

Our results for the contact angles are shown in Fig. 14. In the present case of $J<K, \sigma_{W, G}>\sigma_{A, G}$. Wetting of the water-air interface by the amphiphile-rich phase can now be reached, but it occurs only at sufficiently large $L_{1}$ and $L_{4}$. The interaction parameter $L_{1}$ takes the system towards the critical point, but at the same time reduces $\sigma_{W, G}$. Numerical instabilities prevent us from studying larger values of $L_{1}$ than those shown in Fig. 14. Therefore, wetting of the $W G$ interface by the amphiphile-rich phase has only been found to occur when $\sigma_{W, A}$ is additionally reduced by the $L_{4}$ interactions. The effect of increasing $L_{4}$ can be inferred from the form of Ginzburg-Landau Hamiltonian (23). The term $-L_{4} \rho^{2} \psi^{2}$ has a similar effect as $-C \rho \psi$ since $0<\rho<1$ and 

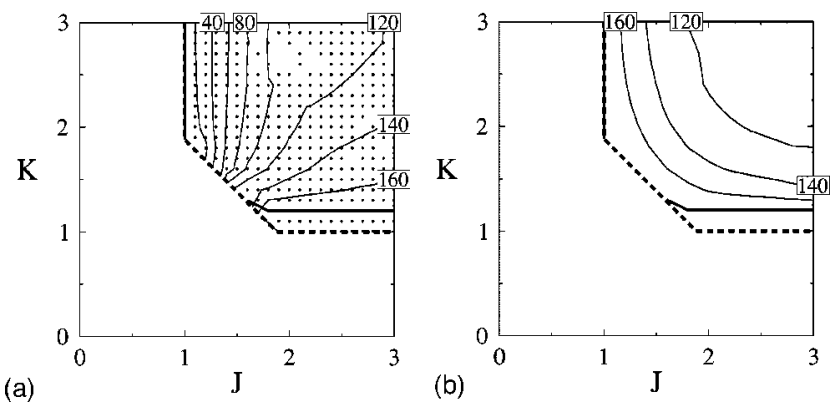

FIG. 15. Cut through the three-phase region for $C=1$, with $L_{1}=0.1$ and $L_{4}=0.025$. Compared to nonamphiphilic mixtures, see Fig. 4, wetting transitions are shifted to higher values of $K$ and $J$, but, as the three-phase region also gets smaller due to an effective shift to higher $C$ by $L_{1}$, the extension of the wetting regions actually declines.

$0<\psi<1$. Therefore, increasing $L_{4}$ has a similar effect as increasing $C$ in the system without amphiphile. For $J<K$, increasing $C$ drives the system of Fig. 7 towards $B$ wetting, which corresponds to wetting by the amphiphile-rich phase in the present case. Physically, the reduction of the interfacial tension $\sigma_{W, A}$ by the interaction $L_{4}$ can be interpreted as the formation of an amphiphilic bilayer, which is favored by $L_{4}>0$; compare Eq. (21).

Figure 15 shows a cut through the three-phase region at $C=1$ with $L_{1}=0.1$ and $L_{4}=0.025$. Compared to a system without amphiphiles (see Fig. 4) the wetting transitions have moved to higher $J$ and $K$, but, as the three-phase region has also become smaller, wetting now occurs closer to the critical end points.

\section{SUMMARY}

The wetting behavior of ternary liquid mixtures has been investigated within a two-order-parameter LandauGinzburg-type model for liquids with short-range interactions, which corresponds to the continuum limit of the Blume-Emery-Griffiths (BEG) model. In the investigated range of interaction parameters, with $0<J<5,0<K<5$, and $0<C \leqslant 2$, all wetting transitions are found to be continuous.

The critical exponent $\gamma$ of the contact angle and the growth exponent $a_{l}$ of the film thickness were calculated both from a numerical solution of the mean-field equations, and within an effective-interfacial-potential approach of independent interfaces. In both cases, we find for a largest part of the wetting diagram a universal wetting behavior with $\gamma$ $=2$ and $a_{l}=1 / \lambda_{1}$. However, near the surfaces $K \simeq 2 J \simeq 2 C$ the wetting exponents become nonuniversal. In particular, they become very large due to a symmetry in the densities of the excess phases' within the wetting film.

The model has then been extended by two interactions which add amphiphilic properties to one of the fluid components. One of the interactions models the amphiphiles' property to assemble at interfaces. It reduces interfacial tensions and therefore supports wetting. In agreement with experimental results, we find that it produces wetting of the microemulsion-air interface by the water phase.

\section{DISCUSSION AND CONCLUSIONS}

\section{A. Comparison with experiments in water-surfactant mixtures}

In the experiments of Kahlweit and Busse, ${ }^{37}$ the wetting behavior of a mixture of water and nonionic surfactant $C_{i} E_{j}$ in coexistence with air was studied as a function of the headgroup size. For $C_{8} E_{0}$ and $C_{8} E_{1}$, the surfactant-rich phase clearly forms a lens at the water-air interface, with a finite contact angle. For $C_{8} E_{2}$ and $C_{8} E_{3}$, on the other hand, the surfactant-rich phase forms a droplet, which hangs at the air-water interface. The reason that the droplet is still attached to this interface is the density difference, which drives the less-dense surfactant-rich phase upwards.

The increase of the head size has the effects (i) of increasing the solubility of the amphiphile in water, and (ii) of increasing the temperature above which two-phase coexistence between water-rich and surfactant-rich phases appears. Since with rising temperature, water becomes an increasingly poorer solvent for the surfactant, these two effects nearly cancel and leave the surfactant concentration of the lower critical point nearly unchanged. ${ }^{38}$ Furthermore, the increase of the head size decreases the tension of the oil-water interface, while the tension of the water-air interface is hardly affected. ${ }^{38}$

The experiments of Ref. 37 have been carried out at a constant of $25^{\circ} \mathrm{C}$. This should be compared with the temperatures of the lower critical point of $T_{c}<0{ }^{\circ} \mathrm{C}$ for $C_{8} E_{0}$, $C_{8} E_{1}, \quad C_{8} E_{2}, \quad T_{c}=11^{\circ} \mathrm{C}$ for $C_{8} E_{3}$ and $T_{c}=40^{\circ} \mathrm{C}$ for $C_{8} E_{4} \cdot{ }^{38}$ Thus, with increasing head-group size, the system at constant temperature effectively approaches a critical point.

In our model, the increased head-group size corresponds to (i) an increase of the interaction parameters $L_{1}$ and $L_{4}$, and (ii) a increase of the coupling constant $C$. The increase of $L_{1}$ has been shown in Fig. 11 to have only a weak effect on the interfacial tension of the liquid-air interface, in agreement with the experimental observations. The increase of $L_{1}$ and the increase of $C$ (compare Sec. II A) both act to move the system closer to a critical point, and thus promote wetting. The theoretical results presented here are therefore in good agreement with the experimental results of Ref. 37.

\section{B. Ternary polymer mixtures}

Ternary systems of particular interest are mixtures of three different polymers of lengths $N_{A}, N_{B}$, and $N_{C}$. In the weak-segregation regime, the polymers are well described by the Flory-Huggins approximation, with interaction parameters $\chi_{\alpha, \beta}$. Then the free energy of a mixture of polymers of equal lengths $N_{A}=N_{B}=N_{C} \equiv N$ takes the form of Eq. (1) with $E^{\alpha, \beta}=-N \chi_{\alpha, \beta}$ for $\alpha \neq \beta, E^{\alpha, \alpha}=0$, and temperature $T$. Mixtures of polymers of different lengths correspond to different temperatures $T_{A}, T_{B}$, and $T_{C}$ as prefactors of the three logarithmic terms in Eq. (3). We have studied such a model for an amphiphilic system also, where the length of water and air molecules was taken to be identical $\left(N_{W}\right.$ $=N_{G}=1$ ), but the amphiphile to be a chain of length $N_{A}$. The qualitative behavior for $N_{A}=10$ was found to be very similar to $N_{A}=1$ in this case. 
Symmetric polymer mixtures, where all the components have the same molecular weight, were studied very recently ${ }^{39}$ in the special case $\chi_{A C}=\chi_{B C}$. The method employed in this study is the Scheutjens-Fleer self-consistentfield theory, in which the polymer conformations are taken into account explicitly in a mean-field approximation. In our model, $\chi_{A C}=\chi_{B C}$ corresponds to $J=K$. The wetting transitions are then studied as a function of $J=2 N \chi_{B C}$ and $J-C$ $=N \chi_{A B}$. In the weak-segregation regime, the gradient term in a binary polymer mixture has the form ${ }^{40}$

$$
\frac{R_{e}^{2}}{36 N} \psi(1-\psi)(\nabla \psi)^{2} \simeq \frac{R_{e}^{2}}{9 N}(\nabla \psi)^{2},
$$

where $R_{e}$ is the end-to-end distance. Thus, the ratio of the amplitudes of the gradient and interaction terms in a Ginzburg-Landau theory of polymeric systems is not the same as in our model. However, it is easy to see that in the mean-field approximation, the amplitude of the gradient term can be absorbed into a rescaling of the coordinate perpendicular to the interface. Therefore, the two models should be equivalent in the weak-segregation regime.

The wetting phase diagram in this parameter space can be reconstructed from Figs. 4, 5, and 6. We find that in the entire range of parameters $0<N \chi_{B C}<2.25$ and $0<N \chi_{A B}$ $<4.5$ investigated in our calculations, the wetting transition is second order, in agreement with the self-consistent-field results of Ref. 39. Not too close to the point where the system is invariant under an exchange of all components, the transition in our model can roughly be approximated by the line $C=0$; compare Fig. 6 . This corresponds to $2 N \chi_{B C}$ $=N \chi_{A B}$ in the polymer model, which approximates the result found in Ref. 39 quite well.

It is most easily seen from Figs. 4 and 5 in combination with Fig. 9 that for the symmetry line $K=2 C$, where the system is invariant under an exchange of $A$ and $C$ molecules, the wetting transition is continuous with nonuniversal wetting exponents. The same behavior must of course occur along the symmetry line $J=K$, where the system is invariant under an exchange of $A$ and $B$ molecules. Therefore, we predict that the wetting transition in the ternary polymer mixture of Ref. 39 should have nonuniversal wetting exponents in the weak segregation regime.

A first-order wetting transition has been observed in Ref. 39 for $N \chi_{A B}>8$. We have therefore investigated the wetting behavior of our model also in the regime of large $J \simeq 8$ (and $C=1$ ) as a function of $K$. The wetting transition is still continuous in our model for these large interaction parameters. It is important to note that values of $N \chi \simeq 10$ no longer fall into the weak-segregation regime, but are in the crossover regime from weak to strong segregation. Therefore, the models should not be expected to be equivalent in this regime.

Another interesting ternary polymer system is the mixture of two homopolymers $A$ and $B$ with an $A B$ diblock copolymer. The diblock copolymer acts as an amphiphile in such mixtures. Indeed, Ginzburg-Landau models for these systems have been derived some time ago, ${ }^{41,42}$ which show the close correspondence of diblock copolymer and amphiphilic systems. This implies that our conclusions about the dependence of the wetting behavior in amphiphilic systems on the chain length of the hydrophilic part should carry over to block copolymer mixtures.

\section{ACKNOWLEDGMENTS}

Support by the Deutsche Forschungsgemeinschaft under Grant No. GO548/3 is gratefully acknowledged. We thank Marcus Müller for helpful discussions about wetting in polymer mixtures.

\section{APPENDIX: SYMMETRY RELATIONS OF THE BEG MODEL}

The BEG model is invariant under an exchange of the $A$ and $B$ components, if the coupling constants $\widetilde{J}, \widetilde{K}$, and $\widetilde{C}$ in Eq. (5) are replaced by $\widetilde{J}^{\prime}=\widetilde{J}, \widetilde{K}^{\prime}=\widetilde{K}$, and $\widetilde{C}^{\prime}=-\widetilde{C}$, respectively. With the relations (7), this implies

$$
J^{\prime}=K, \quad K^{\prime}=J, \quad C^{\prime}=C .
$$

Similarly, the BEG model is invariant under an exchange of the $A$ and $C$ components, if the coupling constants $\widetilde{J}, \widetilde{K}$, and $\widetilde{C}$ in Eq. (5) are replaced by ${ }^{16}$

$$
\begin{aligned}
\widetilde{J}^{\prime} & =(\widetilde{J}+\widetilde{K}+2 \widetilde{C}) / 4, \\
\widetilde{K}^{\prime} & =(9 \widetilde{J}+\widetilde{K}-6 \widetilde{C}) / 4, \\
\widetilde{C}^{\prime} & =(3 \widetilde{J}-\widetilde{K}+2 \widetilde{C}) / 4 .
\end{aligned}
$$

Using again the relations (7), we obtain in this case

$$
\begin{aligned}
& J^{\prime}=J+K-2 C, \\
& K^{\prime}=K, \\
& C^{\prime}=K-C .
\end{aligned}
$$

This implies, in particular, that the plane $C=0$ is mapped onto the plane $K^{\prime}=C^{\prime}$, and vice versa. Over a considerable range of parameters, these planes are very close to the wetting surfaces; compare Figs. 4, 5, and 6.

${ }^{1} \mathrm{~S}$. Dietrich, in Phase Transitions and Critical Phenomena, edited by C. Domb and J. Lebowitz (Academic, London, 1988), Vol. 12.

${ }^{2}$ M. Schick, in Liquids at Interfaces, Proceedings of the XLVIII International Conference, Les Houches, edited by J. Charvolin, J. F. Joanny, and J. Zinn-Justin (Elsevier, Amsterdam, 1990), pp. 417-497.

${ }^{3}$ G. Forgacs, T. M. Nieuwenhuizen, and R. Lipowsky, in Phase Transitions and Critical Phenomena, edited by C. Domb and J. Lebowitz (Academic, London, 1991), Vol. 14.

${ }^{4}$ R. Pandit, M. Schick, and M. Wortis, Phys. Rev. B 26, 5112 (1982).

${ }^{5}$ H. Nakanishi and M. E. Fisher, Phys. Rev. Lett. 49, 1565 (1982).

${ }^{6}$ D. S. Fisher and D. A. Huse, Phys. Rev. B 32, 247 (1985).

${ }^{7}$ K. Binder, D. P. Landau, and D. M. Kroll, Phys. Rev. Lett. 56, 2272 (1986)

${ }^{8}$ K. Binder and D. P. Landau, Phys. Rev. B 37, 1745 (1988).

${ }^{9}$ K. Binder, D. P. Landau, and S. Wansleben, Phys. Rev. B 40, 6971 (1989).

${ }^{10}$ G. Gompper, D. M. Kroll, and R. Lipowsky, Phys. Rev. B 42, 961 (1990).

${ }^{11}$ A. J. Jin and M. E. Fisher, Phys. Rev. B 47, 7365 (1993).

${ }^{12}$ B. Widom, Langmuir 3, 12 (1987).

${ }^{13}$ T. Aukrust and E. Hauge, Phys. Rev. Lett. 54, 1814 (1985).

${ }^{14}$ E. H. Hauge, Phys. Rev. B 33, 3322 (1986).

${ }^{15}$ D. M. Kroll and G. Gompper, Phys. Rev. B 36, 7078 (1987).

${ }^{16}$ S. Dietrich and A. Latz, Phys. Rev. B 40, 9204 (1989).

${ }^{17}$ G. Gompper and M. Schick, in Phase Transitions and Critical Phenomena, edited by C. Domb and J. Lebowitz (Academic, London, 1994), Vol. 16, pp. 1-176. 
${ }^{18}$ M. Kahlweit et al., J. Colloid Interface Sci. 118, 436 (1987).

${ }^{19}$ M. Aratono and M. Kahlweit, J. Chem. Phys. 95, 8578 (1991).

${ }^{20}$ M. Aratono and M. Kahlweit, J. Chem. Phys. 97, 5932 (1992).

${ }^{21}$ G. Gompper and M. Schick, Phys. Rev. Lett. 65, 1116 (1990).

${ }^{22}$ F. Schmid and M. Schick, Phys. Rev. E 49, 494 (1994).

${ }^{23}$ F. Schmid and M. Schick, J. Chem. Phys. 102, 7197 (1995).

${ }^{24}$ K.-V. Schubert, R. Strey, S. R. Kline, and E. W. Kaler, J. Chem. Phys. 101, 5343 (1994).

${ }^{25}$ J. Putz, R. Hołyst, and M. Schick, Phys. Rev. A 46, 3369 (1992).

${ }^{26}$ J. Putz, R. Hołyst, and M. Schick, Phys. Rev. E 48, 635 (1993).

${ }^{27}$ G. Gompper and M. Hennes, J. Chem. Phys. 102, 2871 (1995).

${ }^{28}$ D. Roux et al., Europhys. Lett. 11, 229 (1990).

${ }^{29}$ D. Roux, C. Coulon, and M. E. Cates, J. Phys. Chem. 96, 4174 (1992).
${ }^{30}$ G. Gompper and M. Schick, Phys. Rev. E 49, 1478 (1994).

${ }^{31}$ M. Kahlweit and G. Busse, J. Chem. Phys. 91, 1339 (1989).

${ }^{32}$ M. Blume, V. Emery, and R. Griffiths, Phys. Rev. A 4, 1071 (1971).

${ }^{33}$ D. Furman, S. Dattagupta, and R. Griffiths, Phys. Rev. B 15, 441 (1977).

${ }^{34}$ J. Cahn, J. Chem. Phys. 66, 3667 (1977).

${ }^{35}$ K. Ragil et al., Phys. Rev. Lett. 77, 1532 (1996).

${ }^{36}$ E. Bertrand et al., Phys. Rev. Lett. 85, 1282 (2000).

${ }^{37}$ M. Kahlweit and G. Busse, J. Chem. Phys. 91, 1339 (1989).

${ }^{38}$ M. Kahlweit, G. Busse, and J. Jen, J. Phys. Chem. 95, 5580 (1991).

${ }^{39}$ S. M. Engels and F. A. M. Leermakers, J. Chem. Phys. 114, 4267 (2001).

${ }^{40}$ P.-G. de Gennes, J. Chem. Phys. 72, 4756 (1980).

${ }^{41}$ L. Leibler, Macromolecules 13, 1602 (1980).

${ }^{42}$ R. Hołyst and M. Schick, J. Chem. Phys. 96, 7728 (1992). 